\title{
The New Tapered Fiber Connector and the Test of Its Error Rate and Coupling Characteristics
}

\author{
Qinggui $\mathrm{Hu}^{1}$ and Chengzhong $\mathrm{Li}^{2}$ \\ ${ }^{1}$ Modern Education Technology Center, Neijiang Normal University, Neijiang, Sichuan 641100, China \\ ${ }^{2}$ School of Information Science and Technology, Southwest Jiaotong University, Chengdu 610000, China \\ Correspondence should be addressed to Qinggui Hu; hu646100178@126.com
}

Received 13 December 2016; Accepted 20 March 2017; Published 1 June 2017

Academic Editor: Sulaiman Wadi Harun

Copyright (c) 2017 Qinggui Hu and Chengzhong Li. This is an open access article distributed under the Creative Commons Attribution License, which permits unrestricted use, distribution, and reproduction in any medium, provided the original work is properly cited.

\begin{abstract}
Since the fiber core is very small, the communication fiber connector requires high precision. In this paper, the effect of lateral deviation on coupling efficiency of fiber connector is analyzed. Then, considering the fact that optical fiber is generally used in pairs, one for transmitting data and the other for receiving, the novel directional tapered communication optical fiber connector is designed. In the new connector, the structure of the fiber head is tapered according to the signal transmission direction. In order to study the performance of the new connector, several samples were made in the laboratory of corporation CDSEI and two testing experiments were done. The experiment results show that compared with the traditional connector, for the same lateral deviation, the coupling efficiency of the tapered connector is higher and the error rate is lower.
\end{abstract}

\section{Introduction}

The optical fiber connector is used to realize the interconnection between two optical fibers. As we know, the diameter of multimode fiber is about $50-62.5 \mu \mathrm{m}$. And the diameter of the single-mode fiber is about $8-10 \mu \mathrm{m}$. In order to achieve effective coupling, due to the small diameter of the fiber, the connector requires high precision. This is even true for the single-mode one. Along with the rapid development of communication technology and manufacturing process, the loss caused by the longitudinal and angular deviation has been greatly reduced. But the loss caused by the lateral deviation is still very serious. Especially in some vibration environment, it could cause the communication interruption.

In order to improve coupling efficiency, many scholars have carried out extensive research on the optical transmission theory, manufacturing technology, materials, and so on [1-4]. For example, in 2011, Lambrache and his team [1], at Alef Photonics Center, in Ottawa, Canada, have used the finite difference beam propagation method (FD BPM) to study the refractive index discontinuities in fiber connectors. In 2012, at Central South University of China, Duan and Liu [2] have studied the influence of kinematic variables on apex offset in polishing process of fiber connectors and established the mathematical model of the trajectory end appearing in the polishing motion. In 2013, at NTT Network Laboratory of Japanese, Kihara and his team $[3,4]$ have done the investigation into optical performance of fiber connection with imperfect physical contact and proposed the model of the optical loss mechanism.

In general, those scholars have made great contributions to the application of optical fiber connector. However, most of those studies were concentrated on the connection between the cylindrical fiber heads. In fact, it has inherent flaws relying on the cylindrical heads to achieve coupling. Their common disadvantage is the high precision required. On the other hand, the literatures about the tapered heads are quite few.

As we know, the optical fiber is generally used in pairs, one for transmitting and the other for receiving. In this paper, according to this characteristic, the novel directional tapered fiber connector is put forward. The new connector adopts tapered structure according to the signal transmitting direction. This special structure could reduce the loss caused by the lateral deviation. At the same time, it could also reduce the loss caused by the longitudinal and angular deviation. 


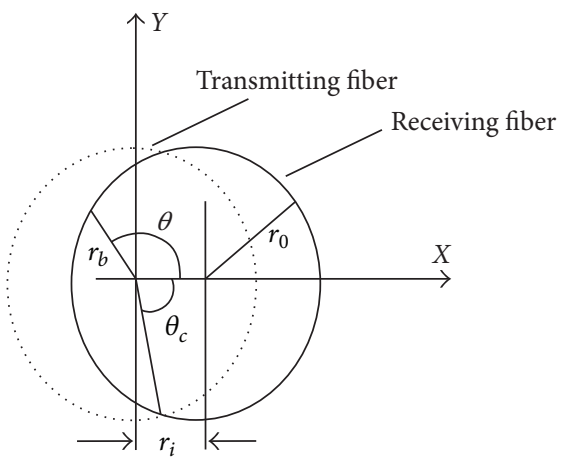

(a) $r_{i}<r_{0}$

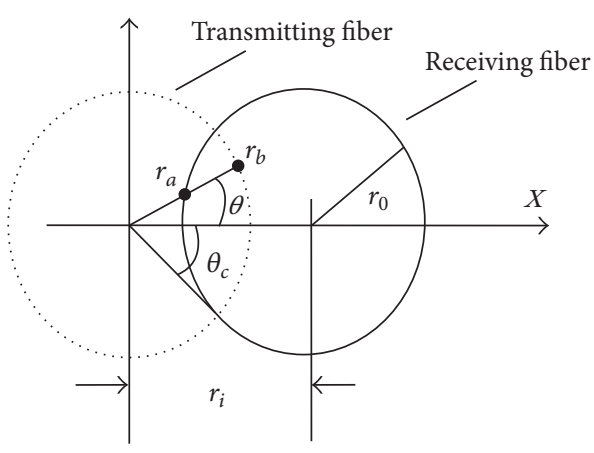

(b) $r_{0}<r_{i}<2 r_{0}$

FIgURE 1: The coordinates of the transmitting and receiving fiber.

\section{The Influence of the Lateral Deviation on the Coupling Efficiency}

The coupling efficiency is an important parameter to measure the characteristics of fiber connectors. Usually, 3 factors could cause the loss of the connector, which are the longitudinal deviation, the angular deviation, and the lateral deviation. As mentioned before, only the loss caused by the lateral deviation is still very serious. Thus, it is necessary to make a deep analysis on it.

Figure 1 shows the lateral deviation between the transmission fiber and the receiving fiber; the lateral deviation between them is $r_{i}$.

As Figure 1 has shown, when the laser is transmitting along $z$-axis in the fiber, the light intensity distribution characteristic could be described by Gaussian beam [4].

$$
I=\left(\frac{A^{2}}{\omega^{2}}\right) \exp \left(-\frac{2 r^{2}}{\omega^{2}}\right) .
$$

In the above formula, $r$ is the distance between the beam spot and $z$-axis, $A$ is the amplitude of the optical beam, and $\omega$ is the beam spot radius; it could be determined by the following formula [5]:

$$
\omega^{2}=\omega_{0}^{2}+\tan ^{2} \theta_{0}\left(z+z_{0}\right)^{2} .
$$

In the above formula, $z_{0}$ is the position of the fiber end face, $z$ is the distance between the output light and the fiber end face, $\omega_{0}$ is the beam waist radius, and $\theta_{0}$ is the divergence angle.

Furtherly, as Figure 1 has shown, the receiving light power $P_{i}$ at the point $\left(x_{i}, y_{i}, z\right)$ is determined by the next formula [6].

$$
P_{i}=\gamma \iint_{s}\left(\frac{A^{2}}{\omega^{2}}\right) \exp \left(-\frac{2 r^{2}}{\omega^{2}}\right) d_{s} .
$$

In the above formula, $s$ is the cross-section of fiber core; it is determined by the formula $s=\pi r_{0}{ }^{2}$, in which $2 r_{0}$ is the fiber core diameter and $\gamma$ is the coupling loss.

In order to make the calculation facilitated, it can be simplified to infinitesimal calculus in one dimension.
When the distance between the transmitting fiber face and the receiving fiber face is neglected, namely, $z=0$, in this case, according to the value of $r_{i}$, which is determined by the formula $r_{i}=\left(x_{i}^{2}+y_{i}^{2}\right)^{1 / 2}$, the receiving power $P_{i}$ can be calculated in two cases.

First case, if $r_{i}<r_{0}$, please see Figure 1(a).

$$
P_{i}=\frac{1}{2} \gamma A^{2} \int_{0}^{\pi}\left[1-\exp \left(-\frac{2 r_{b}}{\omega^{2}}\right)\right] d_{\theta} .
$$

In the above formula, to suppose $\theta_{c}=\arccos \left(r_{i} / 2 r_{0}\right)$, if $0<$ $\theta<\theta_{c}, r_{b}=r_{0}$; if $\theta_{c}<\theta<\pi, r_{b}=r_{i} \cos \theta+\left(r_{0}{ }^{2}-r_{i}{ }^{2} \sin ^{2} \theta\right)^{1 / 2}$. Second case, if $r_{0}<r_{i}<2 r_{0}$, please see Figure 1(b).

$$
\begin{aligned}
P_{i} & =\frac{1}{2} \gamma A^{2}\left\{\int_{0}^{\theta_{c}}\left[\exp \left(-\frac{2 r_{a}^{2}}{\omega^{2}}\right)-\exp \left(-\frac{2 r_{0}{ }^{2}}{\omega^{2}}\right)\right] d_{\theta}\right. \\
& \left.+\int_{\theta_{c}}^{\theta_{d}}\left[\exp \left(-\frac{2 r_{a}^{2}}{\omega^{2}}\right)-\exp \left(-\frac{2 r_{b}^{2}}{\omega^{2}}\right)\right] d_{\theta}\right\} .
\end{aligned}
$$

In the above formula, $r_{a}=r_{i} \cos \theta-\left(r_{0}^{2}-r_{i}^{2} \sin ^{2} \theta\right)^{1 / 2}$; if $\theta_{c}<\arcsin \left(r_{0} / r_{i}\right), \theta_{d}=\arcsin \left(r_{0} / r_{i}\right)$. Otherwise, $\theta_{d}=\theta_{c}$.

On the other hand, the total power of the transmitting fiber $P_{c}$ could be calculated out.

$$
\begin{aligned}
P_{c} & =\iint_{s}\left(\frac{A^{2}}{\omega^{2}}\right) \exp \left(-\frac{2 r^{2}}{\omega^{2}}\right) d_{s} \\
& =\frac{1}{2} \pi A^{2}\left[1-\exp \left(-\frac{2 r_{0}^{2}}{\omega_{c}^{2}}\right)\right] .
\end{aligned}
$$

In the above formula, $\omega_{c}$ is the beam waist radius at the transmitting fiber end face.

Then, the coupling efficiency $\eta$ could be calculated out.

$$
\eta=\frac{P_{i}}{P_{c}} .
$$

To suppose the coupling loss $\gamma$ is 0.95 , the fiber is singlemode fiber, whose diameter is $10.0 \mu \mathrm{m}$. Then, according to different beam spot radius $\omega$, the result of the coupling 


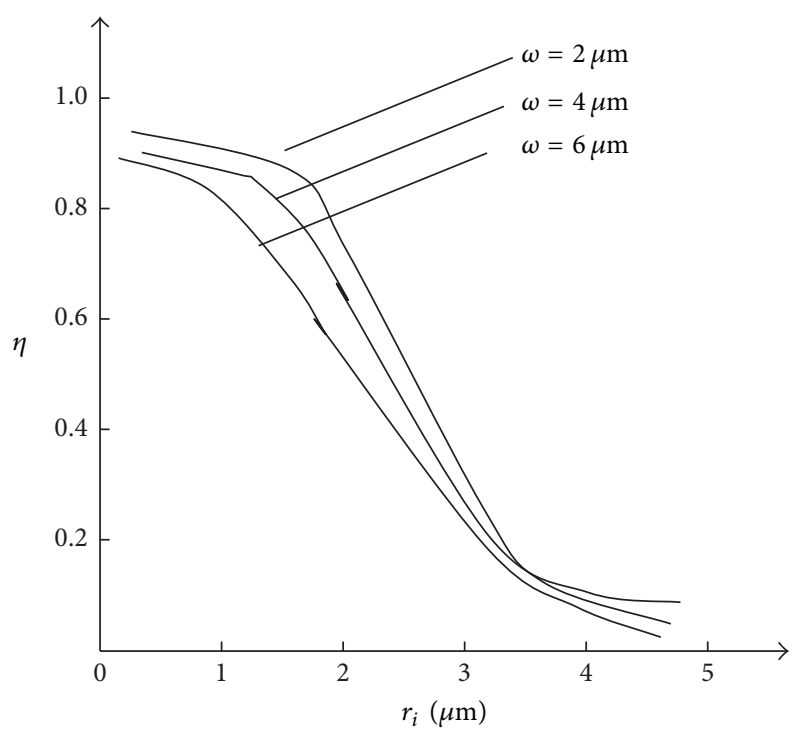

FIgURE 2: The relationship between the coupling efficiency and the lateral deviation $r_{i}$.

efficiency $\eta$ could be calculated out, which is shown as Figure 2.

As shown in Figure 2, the coupling efficiency $\eta$ decreases with the increase of the lateral deviation $r_{i}$. When $\omega>2 \mu \mathrm{m}$, the coupling efficiency $\eta$ begins to drop rapidly.

In fact, $r_{i}$ is the lateral deviation. At present, the loss is mainly caused by it. Then, how to reduce its loss is a key problem. In order to achieve this purpose, the following method is put forward for discussion.

\section{The New Tapered Fiber Connector and the Analysis on Its Total Reflection}

3.1. The New Tapered Fiber Connector. According to the above analysis, we could enlarge the light receiving area to improve the efficiency. The new connector is shown in Figure 3, in which there are 2 sections. One section shows how to connect two fibers. The other shows the different heads of one fiber. In the new connector, the cone bottom receives optical signal. It is the most significant characteristic.

3.2. The Analysis on Its Total Reflection. As shown in Figure 4, it is the tapered structure of the new fiber connector. The refractive index of the fiber core is $n_{1}$. The refractive index of the fiber cladding is $n_{2}$. The refractive index of the surrounding medium is $n_{0}$. The diameter of the cone bottom is $D$. The angle between the inclined plane of the tapered head and the normal of the fiber is $\theta$. Since the material of the tapered cone part is same as that of the fiber core, hence, the refractive index of the tapered cone part is $n_{1}$ too.

As we know, when the beam transmits in the optical fiber, the energy loss will be at minimum on the condition of total reflection [6]. It is the same for the tapered connector.

In order to make the calculation simple, we suppose the light beam enters the tapered connector in the horizontal direction. After the light beam enters the tapered connector, when the light beam reflects at the interface, the angle of incidence is $\beta$. According to the geometric relationship,

$$
\theta=\beta .
$$

To suppose the critical angle of total reflection is $\theta_{c}$, then, we could gain this formula $\theta_{c}=\arcsin \left(n_{2} / n_{1}\right)$. And furtherly, we could gain the following formula:

$$
\theta=\beta \geq \theta_{c}=\arcsin \left(\frac{n_{2}}{n_{1}}\right) .
$$

To suppose the half cone angle of the tapered connector is $\alpha$, then, it should meet the following formula:

$$
\begin{aligned}
\alpha & \leq 90^{\circ}-\theta=90^{\circ}-\beta=90^{\circ}-\theta_{c} \\
& =90^{\circ}-\arcsin \left(\frac{n_{2}}{n_{1}}\right) .
\end{aligned}
$$

The physical meaning of the above formula is that it limits the maximum value of $\alpha$. If it is greater than that value, the total internal reflection will not appear.

\section{The Theoretical Analysis and the Experiment Test}

4.1. The Sample Manufacturing Process. In order to study the performance of the new connector, the author had a discussion with the technicians of Chengdu SEI Optical Fiber Corporation (CDSEI). And what is more, several samples were made in the laboratory of CDSEI. At the same time, the test experiments were done.

Due to the difficulty of producing the new connector directly, the common fiber is reformed with the fused biconical taper (FBT) to gain the new tapered connector. As a welldeveloped manufacturing method, there are a lot of articles discussing its characteristics and coupling mechanism $[7,8]$. In this experiment, we use SCS- 4000 fused taper system to make the samples. In the process of fusing, one single-mode fiber and one other multimode fiber are fused to connect each other. The oxyhydrogen flame is adopted as fusing flame. The range of the fusing flame is set at about $7 \mathrm{~mm}$. The nanoscale quartz powders are adopted as the cobinder.

After the two fibers fused together, the tapered structure appears at the junction. We use the fiber cutting knife to cut the junction to gain the special fiber which has the new tapered head. Then, the wheel type optical fiber polishing machine is adopted to polish the head. For the polishing machine, its grinding accuracy is better than $1 \mu \mathrm{m}$ [9-11]. At last, three samples are made. The cone bottom diameters of all the samples are $15 \mu \mathrm{m}$. Their half cone angles are $6^{\circ}, \mathbf{8}^{\circ}$, and $10^{\circ}$, respectively.

For the new tapered connectors, according to the previous analysis, the maximum half cone angle should be restricted by the following formula:

$$
\begin{aligned}
\alpha & \leq 90^{\circ}-\arcsin \left(\frac{n_{2}}{n_{1}}\right)=90^{\circ}-\arcsin \left(\frac{1.46}{1.49}\right) \\
& =90^{\circ}-78^{\circ}=12^{\circ} .
\end{aligned}
$$




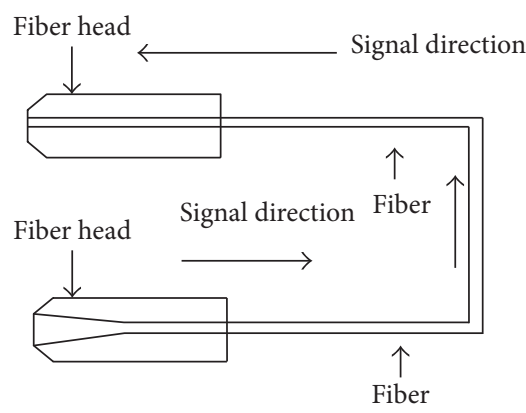

(a) Two heads of one same fiber

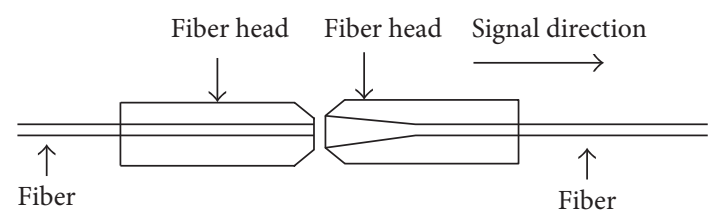

(b) Two fibers connect each other

FIGURE 3: The structure of the directional tapered communication fiber connector.

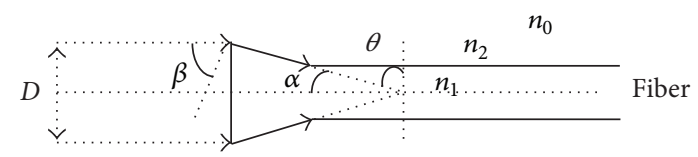

Figure 4: The structure of the new tapered optical fiber connector.

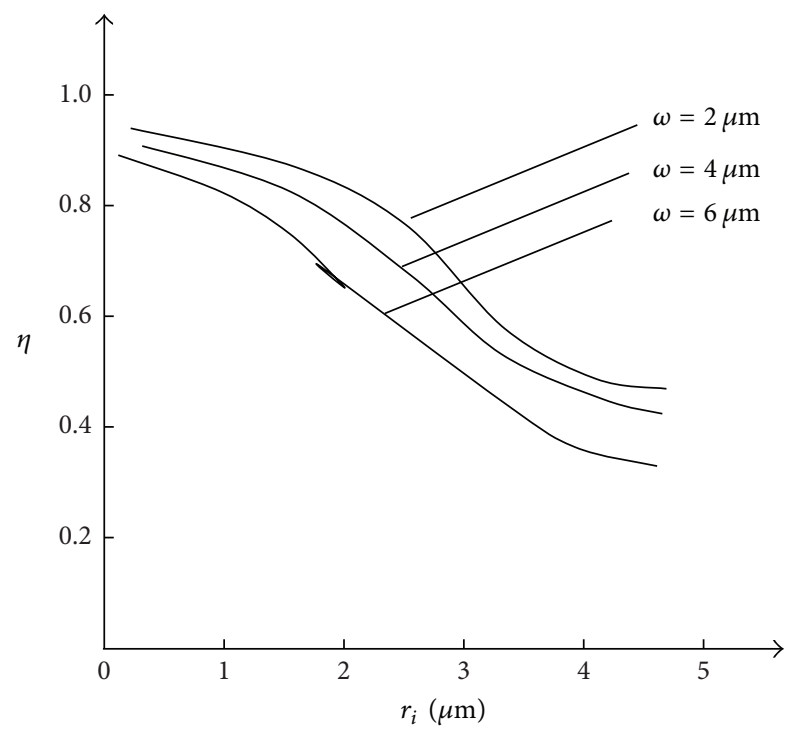

FIGURE 5: The theoretical coupling efficiency of the new fiber connector.

In this experiment, the half cone angles of the new connectors are $6^{\circ}, 8^{\circ}$, and $10^{\circ}$, respectively; they meet the demand.

4.2. The Theoretical Analysis on the Coupling Efficiency of the New Fiber Connector. As mentioned above, for the new tapered connector, the diameter of the cone bottom is about $15.0 \mu \mathrm{m}$. According to theoretical analysis in the second part, for this new connector, $r_{0}$ is equal to $7.5 \mu \mathrm{m}$. We suppose the coupling loss $\gamma$ is 0.95 also. Then, the efficiency $\eta$ could be calculated out, which is shown as Figure 5.

It can be seen from Figure 5 that, compared with the coupling efficiency of the ordinary connector in Figure 2, the efficiency of the new connector is higher. For example, when $r_{i}$ is $5 \mathrm{um}$, coupling efficiency of the new connector is above $40 \%$, while the coupling efficiency of the ordinary connector is below $10 \%$.

4.3. The Coupling Efficiency Measurement Experiment. In order to compare the performance of the new sample with that of the traditional connector, the coupling efficiency measurement experiment is done.

As shown in Figure 6(a), it is the experiment principle. The fixtures are used to fix the fiber. The positions of those fixtures could be adjusted. The camera system could monitor the alignment status of the fibers. Figure 6(b) is the experiment scene.

The light source is a DFB laser. Its threshold current is $12 \mathrm{~mA}$, the operating current is $25 \mathrm{~mA}$, and the max output power is about $5 \mathrm{~mW}$ [9]. In the experiment, the output power is set to $2 \mathrm{~mW}$.

In the experiment, the wave length is $850 \mathrm{~nm} . n_{1}$ is 1.49 and $n_{2}$ is 1.46 ; the influence of $n_{0}$ is ignored. The diameter of the fiber is $10 \mu \mathrm{m}$.

Firstly, the common connector is installed at the position A. And its position is adjusted several times, so that we could gain different lateral deviation. For each adjustment, the power is measured for three times at the position B. Then, the average value is taken. If the optical power measured is $x \mathrm{~mW}$, the coupling efficiency is $\eta=x / 2.00$.

After the coupling efficiency of the common connector is measured, the common connector is replaced by the three new tapered connectors in order. The coupling efficiency of every new connector is measured in the same way. We look at the four connectors including one common connector and the three tapered connectors with the half cone angles of $6^{\circ}, \mathbf{8}^{\circ}, 10^{\circ}$ as CON1, CON2, CON3, and CON4, respectively. Then, the measurement results are shown as in Table 1.

As shown in Table 1, for the common connector, when the lateral deviations $d$ are $1 \mu \mathrm{m}, 2 \mu \mathrm{m}, 3 \mu \mathrm{m}$, and $4 \mu \mathrm{m}$, respectively, the coupling efficiencies are $70 \%, 50 \%, 41 \%$, and $25 \%$ accordingly.

However, for the new tapered connectors CON2, CON3, and CON4, when the lateral deviation $d$ is $1 \mu \mathrm{m}$, the coupling efficiencies are $91 \%, 90 \%$, and $88 \%$ accordingly. When the lateral deviation $d$ is $2 \mu \mathrm{m}$, the coupling efficiencies are $88 \%$, $87 \%$, and $85 \%$. When the lateral deviation $d$ is $3 \mu \mathrm{m}$, the 


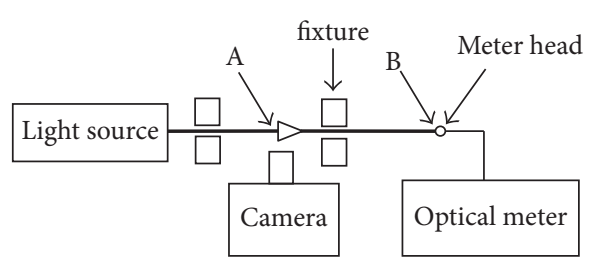

(a) Schematic diagram of the measurement experiment

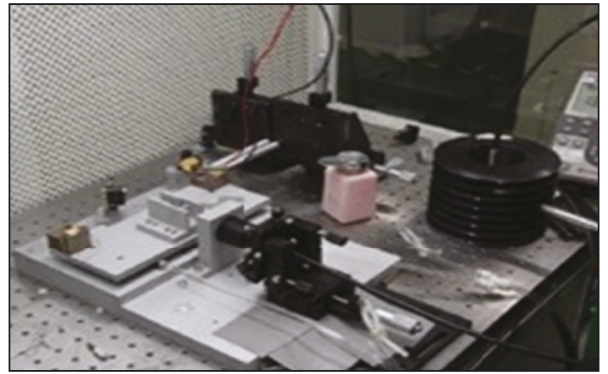

(b) Scene of the measurement experiment

FIGURE 6: The experiment scene of coupling efficiency measurement.

TABLE 1: The coupling efficiency of both the tapered connectors and common connector.

\begin{tabular}{|c|c|c|c|}
\hline Lateral deviation (um) & Connector number & Optic power at position B (mW) & Coupling efficiency \\
\hline \multirow{4}{*}{1} & CON1 & 1.41 & $70 \%$ \\
\hline & CON2 & 1.82 & $91 \%$ \\
\hline & CON3 & 1.81 & $90 \%$ \\
\hline & CON4 & 1.77 & $88 \%$ \\
\hline \multirow{4}{*}{2} & CON1 & 1.01 & $50 \%$ \\
\hline & CON2 & 1.76 & $88 \%$ \\
\hline & CON3 & 1.74 & $87 \%$ \\
\hline & CON4 & 1.71 & $85 \%$ \\
\hline \multirow{4}{*}{3} & CON1 & 0.82 & $41 \%$ \\
\hline & CON2 & 1.50 & $75 \%$ \\
\hline & CON3 & 1.47 & $73 \%$ \\
\hline & $\mathrm{CON} 4$ & 1.45 & $72 \%$ \\
\hline \multirow{4}{*}{4} & CON1 & 0.51 & $25 \%$ \\
\hline & CON2 & 1.31 & $65 \%$ \\
\hline & CON3 & 1.27 & $63 \%$ \\
\hline & CON4 & 1.21 & $60 \%$ \\
\hline
\end{tabular}

coupling efficiencies are $75 \%, 73 \%$, and $72 \%$. When the lateral deviation $d$ is $4 \mu \mathrm{m}$, the coupling efficiencies are $65 \%, 63 \%$, and $60 \%$. The results show the coupling efficiencies of all the tapered connector are higher than that of the common connector.

For the different tapered connectors, the coupling efficiency of CON2 is slightly higher than that of CON3, and the coupling efficiency of CON3 is slightly higher than that of CON4. It means the coupling efficiency of the tapered connectors with the half cone angles of $6^{\circ}$ is highest.

4.4. The Error Rate Measurement Experiment. In order to study the influence of the tapered connector on the quality of data transmission, the experiment of bit error rate test is also carried out. Its basic principle is transmitting data and then receiving it to get the error rate. Error testing technology has been very mature. There are many test instruments for choice. In the experiment, we choose CMR-2048V bit error ratio tester (BERT) to do the experiment, which is the product of Beijing Wangyuan Communication Limited Company. It has a laser transmitter and the wavelength is tunable. Its input

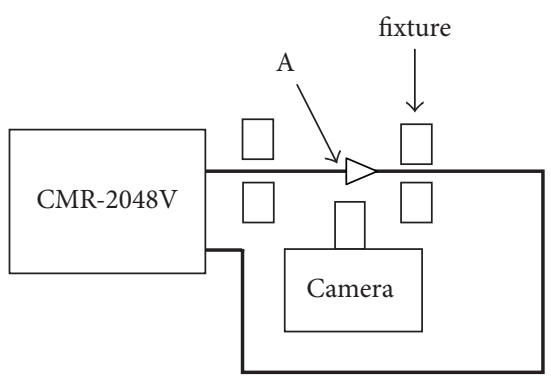

Figure 7: The experiment of bit error rate test.

sensitivity is from $0 \mathrm{~dB}$ to $-43 \mathrm{~dB}$. The bit rate of the digital signal is $2048 \mathrm{~Kb} / \mathrm{s}$. The coding mode is HDB3. Test data frame has two types, which are nonframing and frame. For the frame modes, there are PCM30, PCM30CRC, PCM31, and PCM31CRC for choice $[10,11]$.

As shown in Figure 7, it is the principle of the bit error rate test experiment. The experimental platform is same with that in Figure 6. The difference is that the laser source is replaced 


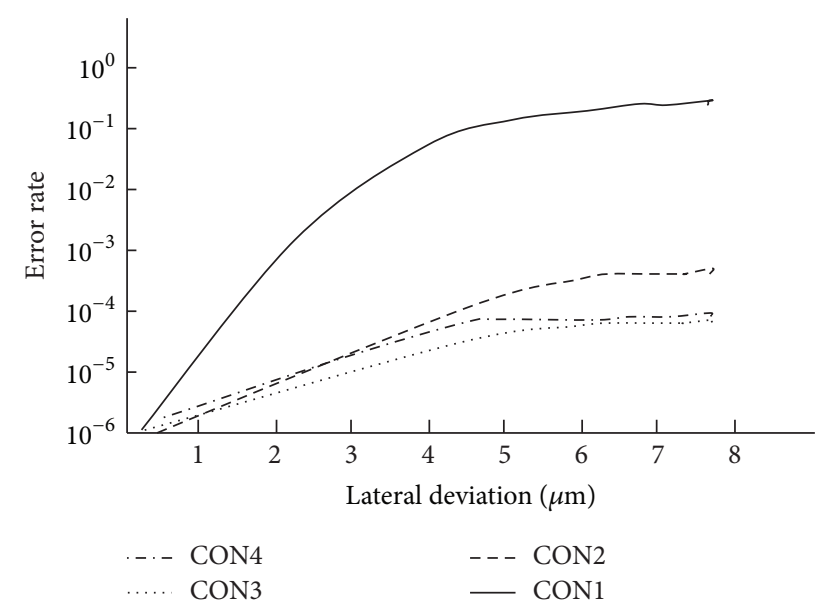

FIgURE 8: The relationship between error rate and lateral deviation (wavelength 850).

by CMR-2048V error tester. The send port and receive port of CMR-2048V connect two ends of the optical fiber.

Firstly, the common connector is installed at the position $\mathrm{A}$ in the fixture. And the fixture is adjusted so that the fiber optic connector could achieve the best alignment state.

The wavelength of the transmitting laser is set to $850 \mathrm{~nm}$. The data flow is set to $100 \mathrm{Mb} / \mathrm{s}$. Then the test starts. The test time lasts 2 minutes. The test data is about $1.2 \times 10^{10} \mathrm{bit}$. After the result is obtained, the fixtures are adjusted so that the lateral deviation is $2 \mu \mathrm{m}, 4 \mu \mathrm{m}, 6 \mu \mathrm{m}, 8 \mu \mathrm{m}, 10 \mu \mathrm{m}, 12 \mu \mathrm{m}$, $14 \mu \mathrm{m}$, and $16 \mu \mathrm{m}$ in order. Every time the fixture is adjusted, the measurement experiment is done in the same way. After the measurement is finished, the fitting curve between the error rate and the lateral deviation is drawn. After that, the wavelength of the transmitting laser is set to $1550 \mathrm{~nm}$, and the measurement experiment is repeated again.

After the measurement experiment of the common connector is finished, it is replaced with the tapered connectors $\mathrm{CON} 2, \mathrm{CON} 3$, and CON4 one by one. Every time the connector is replaced, the measurement experiment is done once more. The final experimental results are shown in Figures 8 and 9.

Both Figures 8 and 9 show that when the lateral deviation increases, the error rate of the common connector increases most rapidly. For example, when the lateral deviation increases to $8 \mu \mathrm{m}$, the error rate of the common connector increases to about $1 \times 10^{-1}$, but all the error rates of the tapered connectors are no more than $1 \times 10^{-4}$.

From Figures 8 and 9, we can see that, for the common connector, the wavelength of the laser has little to do with the relationship between error rate and lateral deviation.

For the three new connectors, when the wavelength is $850 \mathrm{~nm}$, the error rate of CON2 rises the fastest, followed by that of CON4; the error rate of CON3 increases most slowly. When the wavelength is $1550 \mathrm{~nm}$, the error rate of CON4 rises the fastest, followed by that of CON2; the error rate of CON3 increases most slowly. It indicates that the performance of the tapered connector with the half cone angles of $\mathbf{8}^{\circ}$ is best.

On the other hand, in the previous experiment, the coupling efficiency of the tapered connector with the half

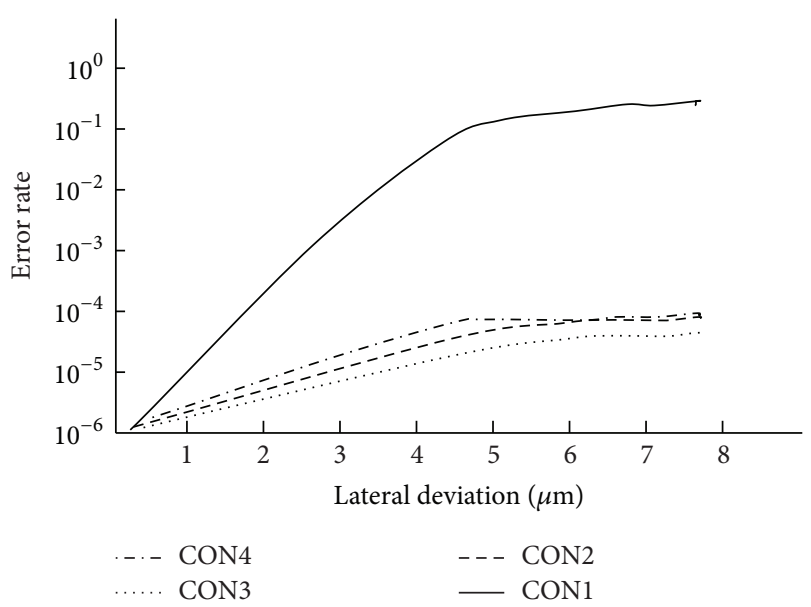

FIGURE 9: The relationship between error rate and lateral deviation (wavelength 1550).

cone angles of $6^{\circ}$ is slightly higher than those of other two. It shows that the different half cone angles have different characteristics. Then, it needs further research on how to choose different tapered connectors in various situations.

\section{The Conclusion}

The optical fiber is generally used in pairs; one of the optical fiber is used for transmitting, and the other is used for receiving. According to this characteristic, the novel directional tapered communication optical fiber connector is designed. The new connector adopts tapered structure according to the signal transmitting direction. This special structure could reduce the loss caused by the lateral deviation. At the same time, it could reduce the loss caused by the longitudinal and angular deviation also. In order to study the performance of the new connector, several samples were made in the laboratory of CDSEI corporation, and two testing experiments were done. The experiment results show that compared with the traditional connector, for the same lateral deviation, the coupling efficiency of the tapered connector is higher and the error rate is lower. The work provides a theoretical reference for the design of the new tapered connector, which could be applied to both the multimode and single-mode optical fiber communication.

\section{Disclosure}

Project has been authorized by Chinese Patent; the name of the patent is "The directional multimode optical fiber with tapered plug"; patent application number is $\mathrm{CN} 201620005088.0$; public/notice number is CN205301623U.

\section{Conflicts of Interest}

The authors Hu Qinggui and Li Chengzhong declare that there are no conflicts of interest regarding the publication of this paper. 


\section{Acknowledgments}

Firstly, the author Qinggui $\mathrm{Hu}$ would like to show his gratitude to his colleague Dr. Long Wen, a respectable and resourceful scholar, who has provided him with guidance in key stage of the writing of this thesis. Without his enlightening instruction, the author could not have completed his thesis. Secondly, the author shall extend his thanks to his wife Dr. Chen Hui for her kindness and help. At last, the author would also like to thank all his other colleagues for their help. Project is supported by the National Natural Science Foundation of China (Grant no. 61275080) and the National Technology Innovation Fund (Grant no. 13C26212201166).

\section{References}

[1] N. Lambrache, P. Pereira, and T. Torobin, "Refractive index discontinuities in fiber optic connectors," Journal of Optoelectronics and Advanced Materials, vol. 13, no. 7, pp. 1010-1014, 2011.

[2] J. Duan and D. Liu, "Influence of kinematic variables on apex offset in polishing Process of fiber optic connectors," Precision Engineering, vol. 36, no. 2, pp. 281-287, 2012.

[3] M. Kihara, M. Uchino, M. Omachi, and H. Watanabe, "Investigation into optical performance of fiber connections with imperfect physical contact," Journal of Lightwave Technology, vol. 31, no. 6, Article ID 6412697, pp. 967-974, 2013.

[4] Z. Tong, W. Han, and Y. Cao, "Fiber sensor for simultaneous measurement of temperature and refraction index based on multimode fiber core-offset," Guangxue Xuebao/Acta Optica Sinica, vol. 34, no. 1, Article ID 0106004, 2014.

[5] V. W. S. Chan, "Optical space communications," IEEE Journal on Selected Topics in Quantum Electronics, vol. 6, no. 6, pp. 959975, 2000.

[6] K. Yang, Q. Hao, and H. Zeng, "All-Optical High-Precision Repetition Rate Locking of an Yb-Doped Fiber Laser," IEEE Photonics Technology Letters, vol. 27, no. 8, pp. 852-855, 2015.

[7] F. Fan, W.-H. Gu, S. Chen, X.-H. Wang, and S.-J. Chang, "State conversion based on terahertz plasmonics with vanadium dioxide coating controlled by optical pumping," Optics Letters, vol. 38, no. 9, pp. 1582-1584, 2013.

[8] Y. Lin, Y. Ai, X. Shan, and M. Liu, "Simulation of twodimensional target motion based on a liquid crystal beam steering method," Optical Engineering, vol. 54, no. 5, Article ID 056102, 2015.

[9] K. Prabu and D. Sriram Kumar, "MIMO free-space optical communication employing coherent BPOLSK modulation in atmospheric optical turbulence channel with pointing errors," Optics Communications, vol. 343, pp. 188-194, 2015.

[10] K. Jung and J. Kim, "All-fibre photonic signal generator for attosecond timing and ultralow-noise microwave," Scientific Reports, vol. 5, Article ID 16250, 2015.

[11] J.-G. Ren, Y.-M. Hu, and X.-L. Zhang, "A united model of fused single-mode fiber coupler," Semiconductor Optoelectronics, vol. 32, no. 1, pp. 18-23, 2011. 

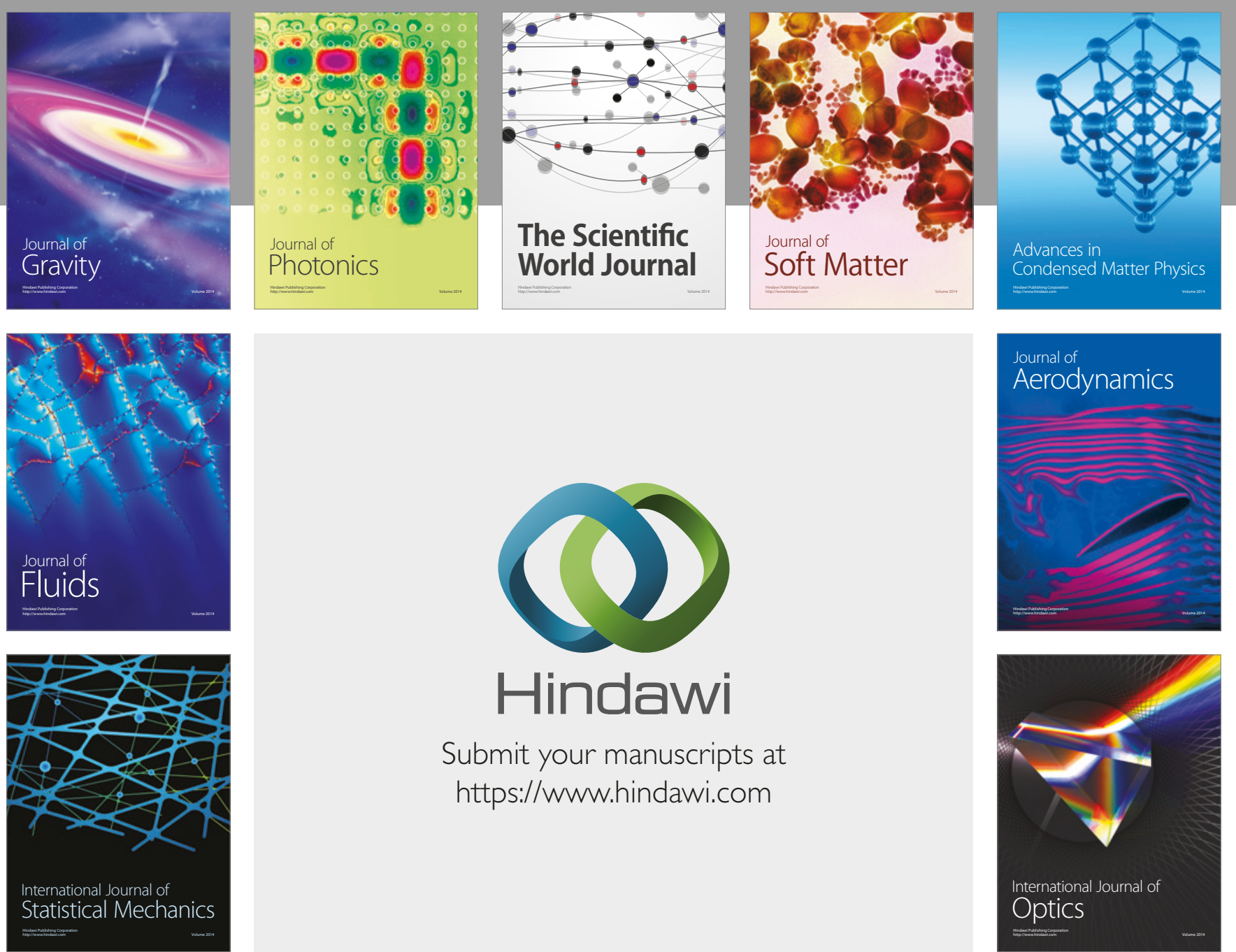

Submit your manuscripts at

https://www.hindawi.com
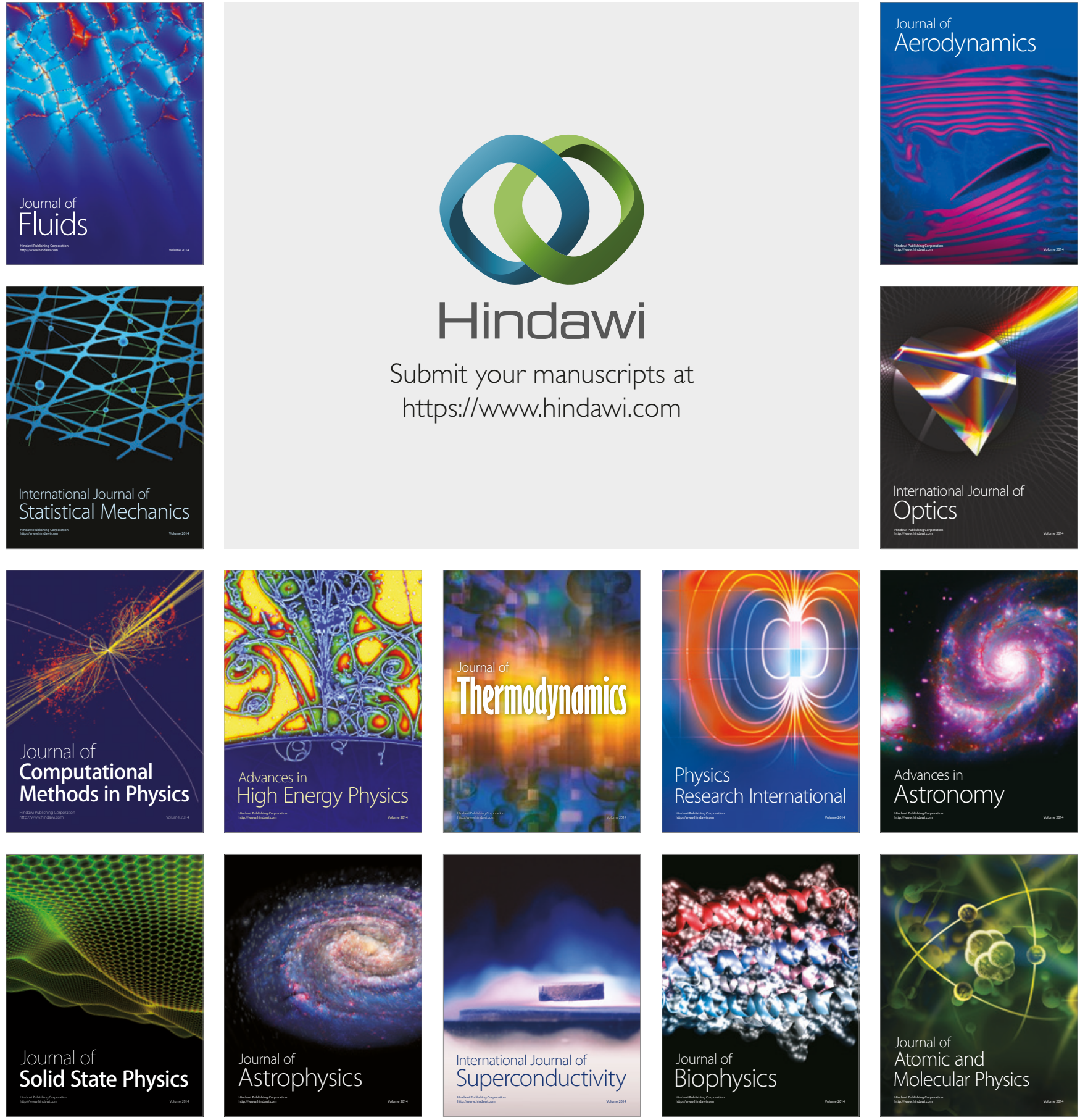